\title{
Research Paper: The Role of Cultural Changes in the Tendency to Childbearing Among Women
}

\author{
Samad Rasoulzadeh Aghdam ${ }^{1}$, Fariba Pourjabbar Akhouni² ${ }^{2}$, Samad Adlipour $^{3^{*}} \mathrm{C}$ \\ 1. Department of Civil Right and Social Based Pathology, Institute for Psycho-Social Health, Azarbaijan Shahid Madani University, Tabriz, Iran. \\ 2. Department of General Psychology, Faculty of Psychology and Education Sciences, Tabriz University, Tabriz, Iran. \\ 3. Faculty of Law and Social Sciences, University of Tabriz, Tabriz, Iran.
}

Citation Rasoulzadeh Aghdam S, Pourjabbar Akhouni F, Adlipour S. The Role of Cultural Changes in the Tendency to Child-
bearing Among Women. Journal of Research \& Health. 2020; 10(2):103-110. http://dx.doi.org/10.32598/JRH.10.2.6
dci" http://dx.doi.org/10.32598/JRH.10.2.6

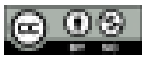

Article info:

Received: 06 Nov 2019

Accepted: 19 Jan 2020

Publish: 01 Mar 2020

\section{Keywords:}

Social Values, Reproductive behavior, Cultural evolution

\section{A B S T R A C T}

Background: Findings and published statistical data show that the fertility rate and the tendency towards childbearing are decreasing in many countries, including Iran. Many personal, social, economic, and cultural factors can cause this decline. Nonetheless, cultural changes have flourished, among other factors in recent years. Therefore, the study aimed to investigate the role of cultural changes in a childbearing tendency among women.

Methods: The research has a correlational design. The statistical population consisted of married women aged 15 to 45 years in Tabriz City, Iran, in 2017. The sample size was calculated as 384 with the Cochran formula. The samples were selected using a multistage cluster sampling method. The study measures were childbearing attitude scale, family values, religious values, global values, and a researcher-made individualism scale. The obtained data were analyzed using the Pearson correlation and multivariate regression.

Results: Findings showed that the tendency towards childbearing of women in Tabriz is low. Besides, there is a significant negative correlation between a tendency towards childbearing and variables of individualism $(r=-0.37, \mathrm{P}=0.000)$ and adherence to universal values $(\mathrm{r}=-0.35$, $\mathrm{P}=0.001$ ). But there is a significant positive relationship between the variables of adherence to family values $(\mathrm{r}=0.32, \mathrm{P}=0.000)$ and religious values $(\mathrm{r}=-0.36, \mathrm{P}=0.000)$ with the tendency towards childbearing. Additionally, independent variables introduced in the research regression model can explain $38 \%$ of the variations in the tendency towards childbearing.

Conclusion: The role of cultural factors affecting procreation should be considered to modify the current reducing trend of childbearing among Iranian families.

\footnotetext{
* Corresponding Author:

Samad Adlipour, PhD.

Address: Faculty of Law and Social Sciences, University of Tabriz, Tabriz, Iran.

Phone: +98 (914) 4244745

E-mail: samadadlipour@gmail.com
} 


\section{Introduction}

I

$\mathrm{n}$ recent years, significant demographic changes have happened all over the world. One of the most influential policies in this regard is applying the birthrate reduction. This policy has remarkably changed the structure of the population and affected the childbearing tendency. In other words, high-level total childbearing has now transferred to the full replacement childbearing or sub-replacement level. This astonishing change has occurred in all countries with different rates and qualities. Iran experienced intensive birthrate control at the end of the $20^{\text {th }}$ century so that in less than a decade, the amount of total childbearing decreased remarkably and in some regions, it reached replacement level. Even in the recent decade, many provinces and urban and rural areas have reported total replacement childbearing and sub-replacement level [1].

Many researchers, policymakers, and even the whole society have shown interest in these significant variations. As a result of these transformations, attitude and tendency toward childbearing have changed [2] and many investigations in Iran and other countries have carried out in this field. In Iran, this matter is mainly studied via the effect of population policies, modernism phenomena, urbanism, education and women's work, but cultural changes in the propensity to childbearing are neglected. Cultural changes are among the most critical variations that Iran's society faced, particularly in post-war years, and it may have a significant role in childbearing reduction [3].

In general, some studies about childbearing reduction have confirmed the effect of such variables as heightening educational level, women's independence, sexual equality $[4,5]$, secularism and individualism [3], media consumption and body management [6], cultural globalization and modern media [7], individualism and body management [8], lifestyle [9, 10], globalization and religiosity [11], cultural pressure and sexual preference [12], modern and traditional values [13]. Some foreign studies insist on parameters like individualism [14], religious prejudices [15], educations [16], worldly modern lifestyle [17], developing mass media, and the culture of consumerism [18].

The changes from collectivism to individualism are some cultural causes for the reduction in childbearing [19]. The popularity of individualism, loss of devotion to family and religious values, and adherence to global values are among effective factors in the propensity to childbearing. The dominance of individualism and be- haviors like living alone, co-tenancy, divorce, and remarriage have decreased the effect of social norms in childbearing [3]. Along with the extensive variations in the society and family, devotion to family and religious values are loosening and breaking down. The changes in these norms have a definitive role in the population trends, including childbearing.

Another research about childbearing and religious values suggests a correlation between the change of religiosity and reduction in childbearing and other related behaviors [4]. Another cultural shift that affects childbearing is the globalization and devotion to these global values, such as sexual equalitarian, a tendency to progress, the negation of fatalism, social rehabilitation, gender attitudes, and attention to the body [20,21]. Nowadays, global values via the mass media, like the Internet and satellite channels, are spreading around the world and promote Western culture, values, and lifestyles in developing countries. So, the modernization of lifestyle and people's attitudes have caused futuristic thoughts and willingness to wealthy life [7]. And this matter, in turn, affects the desire for childbearing.

Regarding the demographic changes in recent decades, it is necessary to clarify policies about childbearing and the population growth rate. Also, considering the effect of social and cultural factors in childbearing, researchers should study the reasons behind women's childbearing behaviors and their determining factors. Accordingly, the present paper investigates the role of cultural changes in the childbearing tendency among married women of Tabriz City, Iran. Out of many cultural factors in childbearing, this study investigates the relationship between individualism and devotion to social values (family, religious, and global values)

\section{Methods}

The research method is correlational, and the study data are gathered by questionnaires in Tabriz in the winter of 2017. The research statistical population consisted of all married women aged 15 to 45 years. The sample size was calculated as 384 with the Cochran formula. The samples were selected using a multistage cluster sampling method. At the first stage, each district of Tabriz City was considered as a cluster and at the second stage, sampling carried out in each cluster and most buildings of each cluster were included in the sampling. At the third stage, final sampling was carried out according to the number of 15- to 45-year-old women in that area and questionnaires were distributed among them and com- 
pleted. For analyzing the research variables, the below scales were used.

\section{The Scale of Childbearing Attitudes}

We used the questionnaire of Rasoolzadeh Aghadam et al. [10] to measure childbearing propensity. This scale involves 9 items that are rated based on a 5-point Likerttype scale from "strongly agree" to "strongly disagree". The reliability of this scale was reported 0.70 in their research. In the present research, the reliability of the scale was estimated as 0.74 by calculating its Cronbach alpha.

\section{The Scale of Devotion to Family Values}

The questionnaire of Rasoolzadeh Aghadam et al. [22] was used to measure this variable. This scale involves 11 items that are rated based on a 5-point Likert-type scale from $5=$ strongly agree to $1=$ strongly disagree. The reliability of this scale was reported 0.76 in their research. In the present research, the reliability of the scale is estimated at 0.74 , which is acceptable.

\section{The Scale of Devotion to Religious Values}

The questionnaire of Rasoolzadeh Aghadam et al. [22] was used to measure this variable. This scale involves 14 items that are rated based on a 5-point Likert-type scale from $5=$ strongly agree to $1=$ strongly disagree. The reliability of this scale was reported 0.72 in their research. In the present research, the reliability of the scale is estimated as 0.82 , which is acceptable.

\section{The Scale of Devotion to Global Values}

To measure this variable, four parameters are considered using Ghanbari Barzban et al. [20] and Haghighatiayn and Darabi studies [21]. These four parameters are social rehabilitation, the tendency to progress and negation of fatalism, sexual equalitarian, and attention to the body. This 35-item scale is rated based on a 5-point Likert-type scale from "strongly agree" to "strongly disagree". The reliability of this scale was reported as 0.78 . The reliability values of its parameters are $0.74,0.75$, 0.72 , and 0.78 for social rehabilitation, the tendency to progress and negation of fatalism, sexual equalitarian, and attention to the body, respectively. Also, the face validity of this scale was suitable according to the opinion of four social experts at Tabriz University.

\section{The Scale of Individualism}

A researcher-made questionnaire was used to measure the individualism variable. This scale is measured using
9 items in the five-point Likert spectrum (from strongly agree to strongly disagree). Two methods of construct validity and face validity were used to validate the items. In this way, after checking the studies done in Iran and other countries, some items were specified about individualism for responders and delivered to social science professors to select the best ones. Then, factor analysis was used for construct validity. In factor analysis, the items which had low factor loading were deleted and final items were determined. Nine items were identified as suitable ones to measure individualism. The total score of the questionnaire ranges from 5 to 45 so that the responder whose overall score is 5-18.33 has low individualism; the responder whose total score is 18.34-31.67 has medium individualism, and the responders whose total score is $31.68-45$ has high individualism. The reliability of items is estimated as 0.74 by calculating the Cronbach alpha.

The research ethical issues were observed by explaining the purpose of this study to responders, keeping the respondents' data confidential, and allowing the responders to leave the study for any reason. The obtained data were analyzed with descriptive statistics (frequency, mean, and standard deviation) and inferential statistics (the Pearson correlation test and multivariate regression) in SPSS V. 21.

\section{Results}

The descriptive findings of this study show that the responders are in the age range of 15 to 45 years, with an average age of 27 years. About $19 \%$ of them are childless, while $44.28 \%$ and $9 \%$ of them have one child, and two or more children, respectively. Another descriptive finding demonstrates that the tendency toward childbearing among married women in Tabriz is lower than the medium level, and they have a low propensity to childbearing.

Table 1 indicates a significant negative relationship between individualism and women's tendency toward childbearing. In other words, with the increase of women's individualism, their propensity for childbearing decreases. Also, there is a significant negative relationship between devotion to global values and the tendency toward childbearing. It has shown that as the devotion to global values increases, the propensity to childbearing decreases. On the other hand, there is a significant direct relationship between women's tendency toward childbearing and devotion to family and religious values. So, their propensity to childbearing would be increased by 
Table 1. The correlation test of predictive variables with women's propensity to childbearing

\begin{tabular}{ccc}
\hline Predictive Variables and Scale & R & Sig. \\
\hline Individualism and tendency toward childbearing & -0.37 & 0.000 \\
\hline Devotion to family values and tendency toward childbearing & 0.32 & 0.000 \\
\hline Devotion to religious values and tendency toward childbearing & 0.36 & 0.000 \\
\hline Devotion to global values and tendency toward childbearing & -0.35 & 0.001 \\
\hline
\end{tabular}

mper

Table 2. The multivariate regression model in women's Propensity to childbearing

\begin{tabular}{|c|c|c|c|c|c|c|c|c|c|}
\hline Independent Variables & B & Beta & Sig. & Tolerance & $\mathbf{R}$ & $\mathbf{R 2}$ & $\mathbf{F}$ & Sig. & Durbin-Watsor \\
\hline Individualism & -0.61 & -0.37 & 0.000 & 0.78 & & & & & \\
\hline Family values & 0.55 & 0.29 & 0.001 & 0.75 & & & & & \\
\hline Religious values & 0.57 & 0.34 & 0.000 & 0.70 & 0.62 & 0.38 & 90.70 & 0.000 & 1.96 \\
\hline Global values & -0.51 & -0.32 & 0.000 & 0.73 & & & & & \\
\hline
\end{tabular}

the increase of women's devotion to family and religious values.

Table 2 presents the multivariate regression model in women's tendency toward childbearing. The multiple correlation coefficient is 0.64 , which demonstrates that the predictive variable has a $64 \%$ simultaneous relationship with women's tendency toward childbearing. Also, the coefficient of determination is 0.38 , which shows that $38 \%$ of the women's tendency to childbearing can be explained with these predictive variables. The standard effect coefficient in Table 2 shows that the most potent predictor of women's childbearing is individualism (- 0.35$)$, devotion to religious values (0.33), devotion to global values (-0.32), and devotion to family values $(0.29)$.

\section{Discussion}

According to the study findings, there is a significant negative relationship between individualism and the tendency to childbearing in married women in Tabriz City. This finding agrees with the results of Rasteghar Khaled and Mohammadi, Mohammadi and Sifori, Sadeghi and Manski, and Mayshar who reported that those with high individualism have a lower propensity to childbearing $[3,14,19,23]$. In this field, Enayat and Parnian found that individualism was related to losing traditions and cultural customs. This phenomenon is related to modernity and globalization.
Modernity, due to its humanism essence, emphasizes the human's maximum utilization from mundane things. Thus, some traditions with basic values in Iran's folk culture, such as family relationships, collectivism, having more children, extended family, etc., are significantly affected by the penetration of Western values and replaced with such new values as individualism. Under the effect of this discourse, people are looking for progress, independence, and freedom in different fields and more children are considered as an obstacle to individual development [7].

Also, according to this view, children are imposing more costs in life, and based on capitalism in modernity, childbearing does not have any economic explanation. On the other hand, the basis of modernism view is high comfort and pleasure. With this point of view, the motto of (more children, better life) gets humanism and individualism interpretation. Now, the child's care and other limitations which children make for parents are considered as an obstacle to their convenience and comfort. So childbearing is regarded as a negative and disturbing phenomenon in life [24].

According to our study results, there is a negative relationship between the devotion to family values and a tendency to childbearing. Thus, as the devotion of women to family values increases, their propensity to childbearing increases. The variation in the family system is one of the significant parameters in the field of childbearing. 
The formation of the nucleus and single-parent families (because of divorce) instead of the extended families are among these variations. Davis and Blake believe that the proponents of birthrate increase are more common in extended families than nucleus families because children are considered as an economic blessing, and they are working power of a family. Also, they can support their parents in old ages [25].

Other variations in family systems can be seen, including reduction of marriage, an increase in the average marriage age, an increase in the divorce rate, and an increase in co-tenancy. These factors have changed the model and tendency of childbearing. These variations in family systems have attenuated the devotion to family values. On the other hand, the devotion to family values like the blessing of marriage, marriage in younger ages, disapproval of divorce, the importance of motherhood, etc., are the main reasons for childbearing.

Other findings of this study showed a significant direct relationship between the devotion to religious values and a tendency to childbearing. This finding agrees with the results of Rasteghar Khaled and Mohammadi, Piltan and Rahmanian, Kalantari et al., Raad and Savabi, and Adhikari [3, 26-29]. As religious teachings preach forming the family, banning sexual relations before marriage, encouraging pregnancy, forbidding abortion, and having more children, it is natural that those with high devotion to religious values have a higher propensity to childbearing.

Another finding of this study showed a significant there is a negative relationship between the devotion to global values (such as sexual equalitarian, a tendency to progress and negation of fatalism, social rehabilitation, attention to the body), and tendency to childbearing. This finding is consistent with the results of Mohmodian et al., Eshaghi et al., Ghodrati et al., and Naghdi and Zare [6, 30-32]. Nowadays, cultural diffusion of global values in many countries has led to the modernism of lifestyle, people's thoughts and attitudes, growth of futurism, and more comfortable life, so that these parameters, in turn, have attenuated human's attention to the family. In other words, with the extension of global values, such as individualism, comfort, profiteering, selfish convenience, social rehabilitation in motherhood, social rehabilitation in child value, the importance of personal progress via profession and education, the importance of health and body fitness for girls and woman are being publicized all over the world.

Moreover, Giddens believed that the roles of manhood and womanhood, which were respectively breadwinning and householding, have changed in modern societies. He thought that manhood and womanhood, in terms of duty and social identity, do not have any apparent meanings. Nowadays, men and women are living not according to their pre-determined roles, and women are more sexual equalitarian than before. In other words, in the modern era, such values as faithfulness and sympathy between spouses, childbearing and other sexual roles like breadwinning, householding, and children breeding have weakened seriously and the family lacks its previous sacredness anymore. So women are not obligated to childbearing and its upbringing and they consider new roles for themselves for individual advancement. In other words, women take other roles beyond their private, motherhood and spouse roles and they have a wide variety of identity-maker sources [7].

The regression model of this study suggests that individualism, devotion to religious values, devotion to global values, and devotion to family values have the most influence on the propensity to childbearing in married women in Tabriz, respectively. In this field, the findings of Rasteghar Khaled and Mohammadi and Enayat and Parnian showed that individualism, religiosity, the diffusion of global values via modern technologies, and devotion to family values have a significant effect on the propensity to childbearing in families $[3,7]$.

It should be mentioned that all data are collected via questionnaires, and despite enough explanations about the study, the mood and spirit of responders could affect their answers. The study population consisted of married women in Tabriz aged 15 to 45 years and older women were not enrolled in this study because it increased the study cost. We suggest that other researchers add the data of pre-revolution women generation in future studies. Furthermore, the result of this investigation is not generalizable to rural women because it was carried out on urban women.

\section{Conclusions}

Cultural factors, besides social and economic ones, have a remarkable part in the lack of propensity to childbearing in married women. So, their tendency to childbearing decreases as the individualism, and the devotion to global values increases. Also, their devotion to family and religious values has an effective role in the reduction of propensity to childbearing among 15- to 45-year-old women. These results can show the perspective of the current situation of Iranian women's childbearing and can be a guideline for programmers in making short-, medium- and long-term policies toward childbearing. 
The role of cultural factors, like individualism and the devotion to family, global and religious values, should be considered to correct the current condition and plan to change it.

\section{Ethical Considerations}

\section{Compliance with ethical guidelines}

This article has received Ethical Approval (No. 318/d/28362) and registered on December 24, 2019.

\section{Funding}

The author(s) received no financial support for the research, authorship and/or publication of this article.

\section{Authors' contributions}

Study design: Samad Rasoulzadeh Aghdam, Samad Adlipour; Data collection and analysis: Fariba Pourjabbar Akhouni, Samad Adlipour; Manuscript preparation: All authors and All authors have read and approved the final version.

\section{Conflict of interest}

The authors declared no conflict of interest.

\section{References}

[1] Mahmoudian H, Rezaei M. Women, and low-propagation (case study of Kurdish women). Women's Strateg Stud. 2012 14(55):173-225.

[2] Kalantari S, Rabbani R, Aktaei R. The study of social, economic and cultural factors affecting the fertility rate: Emphasizing the role of family planning in Isfahan. J Soc Sci. 2006; 1(1):107-50.

[3] Rastegar Khaled A, Mohammadi M. Cultural changes and fertility decline in Iran (based on secondary analysis of the data of the survey of Iranian values and attitudes). Appl Sociol. 2015; 26(58):159-80.

[4] Abbasi Shawazi MJ, Alimandgari M. The effect of different dimensions of women's independence on their fertility behavior in Iran. J Women's Res. 2010; 8(28): 31-51.

[5] Abbasi Shawazi MJ, McDonald P, HosseiniChavoshi M. The fertility transition in Iran: Revolution and reproduction New York: Springer, 2009. [DOI:10.1007/978-90-481-3198-3] [PMID] [PMCID]

[6] Mahmoudian H, KochaniEsfahani M, Moghaddas S. Media consumption, body management and fertility behaviors (Case study: Female teachers in Yasouj). J Culture Strateg. 2015; 8(31):174-96.
[7] Enayat H, Parnian L. Study of the relationship between cultural globalization and the propensity for childbearing. Woman and Soc Q. 2013; 4(2):109-36.

[8] Chamani S, Shakribii A, Moshfegh M. Sociological determinants of childbearing (case study: Married women in Tehran). Sociocult Strateg Q. 2016; 5(20):137-65.

[9] KavehFiruz Z, Zareh B, Shamsoddini H. The Effect of Lifestyle Components on Attitude towards Childbearing (case study: Women at the eve of marriage of Tehran). Woman in Development and Politics. 2016; 14(2):217-34.

[10] Rasoolzadeh Aghdam S, Afshar S, Adlipour S, Mir Mohammadtabar SA. Analysis of the relationship between social capital and lifestyle with tendency to childbearing (case study: Students of azerbaijan Shahid Madani University). Sociocult Strateg Q. 2017; 5(20):107-35.

[11] Ojaghlou S, Moradi A, Ghasemi I, Arshad J. Generational changes in the value of children and the childbearing behavior of women before and after the Islamic Revolution (case study: Zanjan City). Woman in Cult and Art. 2012; 6(2):40925.

[12] Pezhhan A, Kamaliha A. The effect of cultural factors on women's reproduction in $15-50$ years of the $12^{\text {th }}$ district of Tehran. Cult Socio Res Q. 2015; 6(2):115-37.

[13] Seyedmirizai SM, Arshadi H. A comparison of the effect of traditional and modern values on the propensity to fertility in two generations in sanandaj (1343-1363, 1364-1378). J Iran Soc Sci Stud. 2015; 12(45):64-86.

[14] Manski C, Mayshar J. Private incentives and social interactions: Fertility puzzles in Israel. J Eur Econ Assoc. 2003; 4(2):181-211. [DOI:10.1162/154247603322256800]

[15] Frejka T, Westoff CF. Religion, religiousness and fertility in the US and in Europe. Rostock: Max Planck Institute for Demographic Research; 2006. [DOI:10.4054/MPIDRWP-2006-013]

[16] Edwards ME. Education and occupations: Reexamining the Conventional wisdom about later first births among American mothers. Sociol Forum. 2002; 17(3):423-43. [DOI:10.1023/A:1019679023616]

[17] Kertzer D, White M, Bernardi L, Gabrielli G. Italy's path to very low fertility: The adequacy of economic and second demographic transition theories. Eur J Popul. 2009; 25(1):89-115 [DOI:10.1007/s10680-008-9159-5] [PMID] [PMCID]

[18] Murthi M. Fertility change in Asia and Africa. World Dev. 2003; 30(10):1769-78. [DOI:10.1016/S0305-750X(02)00062-1]

[19] Sadeghi R. Analysis of socio-cultural context of fertility decline in Iran. Soc Cult Strateg. 2016; 5(20):217-46.

[20] GhanbariBarzian A, Jalayipour HR, Shaverdi T. [Acceptance of world values among Arabic-speaking Iranian (Arabs of Khuzestan Province) (Persian)]. J Iran Soc Stud Res. 2012 1(3):135-58.

[21] Haghighatian M, Darabi M. Investigating the impact of cultural modernization and its impact on family values (case study: Nahavand county). Sociol Stud Youth. 2015; 36(20):5788 [Persian].

[22] Rasoulzadeh Aghdam S, Mir Mohammad Tabar SA, Afshar S, Adlipour S. Sociological analysis of the consequences 
of social media on the values of Iranian youth. Sociocult Strateg Q. 2015; 5(17):65-94.

[23] Mohammadi N, Sayfouri B. Sociological study of factors affecting women's fertility preference. Cultural-Educational of Women Fam Q. 2016; 11(36):49-70.

[24] Jokar M. Modernity, lifestyle change and population decrease in Iran. Journal of Res on Islam Women's and Fam Stud. 2014; 2(2):39-69.

[25] Hosseini H. Economic Sociology and Family Planning. Hamadan: HamadanUniversity; 2011.

[26] Piltan F, Rahmanian M. Sociological study of factors affecting the tolerance of children of married women and men (case study: Women and men aged 25-45 years in Jahrom. J Iran Soc Dev Stud. 2015; 7(2):121-34.

[27] Kalantari S, Abbaszadeh M, Mozaffari F, RakaeiBonab N Sociological study of tendency to childbearing and some related factors (case study: Married youth of Tabriz City). Appl soc. 2010; (37):83-104.

[28] Rad F, Savabi H. Investigating the tendency towards child bearing and its related factors (case study of married women 15-15 years living in Tabriz city). Women and Fam Stud. 2015; 3(1):127-55.

[29] Adhikari R. Demographic, socio-economic, and culturalfactors affecting fertility differentials in Nepal. BMC Pregnancy Childbirth. 2010; 10(19):3-11. [DOI:10.1186/1471-239310-19] [PMID] [PMCID]

[30] Eshaghi M, Mohebbi SF, Papinejad S, Jahandar Z. Working women's childbearing challenges: A qualitative study. Woman in Development And Politics. 2014; 12(1):111-34.

[31] Ghodrati H, Bostani D, Ghodrati A, KalaliSA. The study of relationship between gender equality in family and women's fertility (case study: married women 20 to 40 years old in Sabzevar). J Arid Geogr Stud. 2013; 3(11):75-91.

[32] Naghdi A, Zareh S. The study of social and cultural factors affecting the fertility of marginalized women. Woman and Soc Q. 2013; 4(2):31-54. 
This Page Intentionally Left Blank 